\title{
RACSAMI
}

Rev. R. Acad. Cien. Serie A. Mat.

VOL. 103 (1), 2009, pp. 11-15

Análisis Matemático / Mathematical Analysis

\section{Weighted composition operators between weighted Bergman spaces}

\section{Elke Wolf}

\begin{abstract}
We study the boundedness of weighted composition operators acting between weighted Bergman spaces.

\section{Operadores de composición ponderados entre espacios de Bergman con} pesos
\end{abstract}

Resumen. Se estudia la acotación de los operadores de composición ponderados entre espacios de Bergman con pesos.

\section{Introduction}

We consider strictly positive bounded continuous functions (weights) $v$ and $w$ on the open unit disk $D$ in the complex plane. Moreover let $H(D)$ denote the space of all holomorphic functions on $D$ and let $\phi$ be an analytic self map of $D$ as well as $\psi: D \rightarrow \mathbb{C}$ be analytic. Such maps induce a linear weighted composition operator $\psi C_{\phi}(f)=\psi(f \circ \phi)$. We are interested in weighted composition operators acting on weighted Bergman spaces

$$
A_{w}^{p}:=\left\{f \in H(D) ; \quad\|f\|_{w, p}=\left(\int_{D}|f(z)|^{p} w(z) \mathrm{d} A(z)\right)^{\frac{1}{p}}<\infty\right\}, \quad 1 \leq p<\infty,
$$

where $\mathrm{d} A(z)$ is the area measure on $D$ normalized so that area of $D$ is 1 . Thus $A_{1}^{2}$ denotes the usual Bergman space. An introduction to the concept of Bergman spaces is given in [7] and [8]. Composition operators and weighted composition operators have been studied on various spaces of holomorphic functions, see e.g. [10, 9, 1, 2, 3, 4, 12]. For more general information on composition operators we refer to the monographs [5] and [11]. In this article we want to charaterize boundedness of composition operators acting between weighted Bergman spaces.

\section{Preliminaries}

For $a, z \in D$ let $\sigma_{a}(z)$ be the Möbius transformation of $D$ which interchanges 0 and $a$, that is

$$
\sigma_{a}(z)=\frac{a-z}{1-\bar{a} z}
$$

Presentado por / Submitted by José Bonet Solves.

Recibido / Received: 24 de noviembre de 2008. Aceptado / Accepted: 14 de Enero de 2009.

Palabras clave / Keywords: Weighted Bergman space, composition operator.

Mathematics Subject Classifications: 47B33, 47B38.

(C) 2009 Real Academia de Ciencias, España. 
Furthermore we use the fact that

$$
-\sigma_{a}^{\prime}(z)=\frac{1-|a|^{2}}{(1-\bar{a} z)^{2}}, \quad z \in D
$$

Moreover let $K_{a}(z)=\frac{1}{(1-\bar{a} z)^{2}}$ denote the Bergman kernel and $k_{a}(z)=-\sigma_{a}^{\prime}(z)=\frac{1-|a|^{2}}{(1-\bar{a} z)^{2}}=(1-$ $\left.|a|^{2}\right) K_{a}(z)$ the normalized Bergman kernel in $A_{1}^{2}$ so that $\left\|k_{a}\right\|_{1,2}=1$. For an analytic self map $\phi$ of $D$ and weights $v, w$ on $D$ we define the weighted $(\phi, v)$-Berezin transform of $w$ as follows

$$
\left[B_{\phi, v}(w)\right](a)=\int_{D}\left|\sigma_{a}^{\prime}(\phi(z))\right|^{2} \frac{w(z)}{v(\phi(z))} \mathrm{d} A(z)
$$

In order to find results on composition operators acting on weighted Bergman spaces we need the Carleson measure. To use this we collect some facts. Let $\mu$ be a positive Borel measure on $D$. Then $\mu$ is called a Carleson measure on the Bergman space if there is a constant $C>0$ such that, for any $f \in A_{1}^{2}$

$$
\int_{D}|f(z)|^{2} \mathrm{~d} \mu(z) \leq C\|f\|_{1,2}^{2} .
$$

For an arc $I$ in the unit circle $\partial D$ let $S(I)$ be the Carleson square defined by

$$
S(I)=\left\{z \in D ; \quad 1-|I| \leq|z|<1, \quad \frac{z}{|z|} \in I\right\} .
$$

The following result is well-known. In its present form it is taken from [6] (see there Theorem A).

Theorem 1 ([6, Theorem A]) Let $\mu$ be a positive Borel measure on D. Then the following statements are equivalent.

(i) There is a constant $C_{1}>0$ such that for any $f \in A_{1}^{2}$

$$
\int_{D}|f(z)|^{2} \mathrm{~d} \mu(z) \leq C_{1}\|f\|_{1,2}^{2} .
$$

(ii) There is a constant $C_{2}>0$ such that, for any arc $I \in \partial D$,

$$
\mu(S(I)) \leq C_{2}|I|^{2} .
$$

(iii) There is a constant $C_{3}>0$ such that, for every $a \in D$,

$$
\int_{D}\left|\sigma_{a}^{\prime}(z)\right|^{2} \mathrm{~d} \mu(z) \leq C_{3} .
$$

In the sequel we consider the following weights. Let $\nu$ be a holomorphic function on $D$, non-vanishing, strictly positive on $\left[0,1\right.$ and satisfying $\lim _{r \rightarrow 1} \nu(r)=0$. Then we define the weight $v$ as follows $v(z)=$ $\nu\left(|z|^{2}\right)$ for every $z \in D$.

Next, we give some illustrating examples of weights of this type:

(i) Consider $\nu(z)=(1-z)^{\alpha}, \alpha \geq 1$. Then the corresponding weight is the so-called standard weight $v(z)=\left(1-|z|^{2}\right)^{\alpha}$.

(ii) Select $\nu(z)=\mathrm{e}^{-\frac{1}{(1-z)^{\alpha}}}, \alpha \geq 1$. Then we obtain the weight $v(z)=\mathrm{e}^{-\frac{1}{\left(1-|z|^{2}\right)^{\alpha}}}$.

(iii) Choose $\nu(z)=\sin (1-z)$ and the corresponding weight is given by $v(z)=\sin \left(1-|z|^{2}\right)$.

For a fixed point $a \in D$ we introduce a function $v_{a}(z):=\nu(\bar{a} z)$ for every $z \in D$. Since $\nu$ is holomorphic on $D$, so is the function $v_{a}$. 


\section{Boundedness}

We first need the following auxiliary result. The following lemma is well-known for standard weights (see [7] or [8]) but to the best of our knowledge not known for the weights described above.

Lemma 1 Let $v$ be a radial weight as defined in the previous section (i.e. $v(z):=\nu\left(|z|^{2}\right)$ for every $z \in D$ ) such that $\sup _{a \in D} \sup _{z \in D} \frac{v(z)\left|v_{a}\left(\sigma_{a}(z)\right)\right|}{v\left(\sigma_{a}(z)\right)} \leq C<\infty$. Then

$$
|f(z)| \leq \frac{C^{\frac{1}{p}}}{v(0)^{\frac{1}{p}}\left(1-|z|^{2}\right)^{\frac{2}{p}} v(z)^{\frac{1}{p}}}\|f\|_{v, p}
$$

for all $z \in D, f \in A_{v, p}$.

PROOF. Let $\alpha \in D$ be an arbitrary point. Consider the map

$$
T_{\alpha}: A_{v}^{p} \rightarrow A_{v}^{p}, \quad T_{\alpha}(f(z))=f\left(\sigma_{\alpha}(z)\right) \sigma_{\alpha}^{\prime}(z)^{\frac{2}{p}} v_{\alpha}\left(\sigma_{\alpha}(z)\right)^{\frac{1}{p}} .
$$

Then a change of variables yields

$$
\begin{aligned}
\left\|T_{\alpha} f\right\|_{v, p}^{p} & =\int_{D} v(z)\left|f\left(\sigma_{\alpha}(z)\right)\right|^{p}\left|\sigma_{\alpha}^{\prime}(z)\right|^{2}\left|v_{\alpha}\left(\sigma_{\alpha}(z)\right)\right| \mathrm{d} A(z) \\
& =\int_{D} \frac{v(z)\left|v_{\alpha}\left(\sigma_{\alpha}(z)\right)\right|}{v\left(\sigma_{\alpha}(z)\right)}\left|f\left(\sigma_{\alpha}(z)\right)\right|^{p}\left|\sigma_{\alpha}^{\prime}(z)\right|^{2} v\left(\sigma_{\alpha}(z)\right) \mathrm{d} A(z) \\
& \leq \sup _{z \in D} \frac{v(z)\left|v_{\alpha}\left(\sigma_{\alpha}(z)\right)\right|}{v\left(\sigma_{\alpha}(z)\right)} \int_{D}\left|f\left(\sigma_{\alpha}(z)\right)\right|^{p}\left|\sigma_{\alpha}^{\prime}(z)\right|^{2} v\left(\sigma_{\alpha}(z)\right) \mathrm{d} A(z) \\
& \leq C \int_{D} v(t)|f(t)|^{p} \mathrm{~d} A(t)=C\|f\|_{v, p}^{p} .
\end{aligned}
$$

Now put $g(z)=T_{\alpha}(f(z))$. By the mean-value property we obtain

$$
v(0)|g(0)|^{p} \leq \int_{D} v(z)|g(z)|^{p} \mathrm{~d} A(z)=\|g\|_{v, p}^{p} \leq C\|f\|_{v, p}^{p} .
$$

Hence

$$
v(0)|g(0)|^{p}=v(0)|f(\alpha)|^{p}\left(1-|\alpha|^{2}\right)^{2} v(\alpha) \leq C\|f\|_{v, p}^{p} .
$$

Thus $|f(\alpha)| \leq C^{\frac{1}{p}} \frac{\|f\|_{v, p}}{v(0)^{\frac{1}{p}}\left(1-|\alpha|^{2}\right)^{\frac{2}{p}} v(\alpha)^{\frac{1}{p}}}$. Since $\alpha$ was arbitrary, the claim follows.

Thus, we can give the following sufficient condition for the boundedness of an operator $\psi C_{\phi}: A_{v}^{p} \rightarrow A_{w}^{p}$.

Proposition 1 Let $w$ be a weight and $v$ be a weight as in Lemma 1. If

$$
\sup _{z \in D} \frac{|\psi(z)| w(z)^{\frac{1}{p}}}{\left(1-|\phi(z)|^{2}\right)^{\frac{2}{p}} v(\phi(z))^{\frac{1}{p}}}<\infty
$$

then the operator $\psi C_{\phi}: A_{v}^{p} \rightarrow A_{w}^{p}$ is bounded.

Proof. Applying Lemma 1 we get for every $f \in A_{v}^{2}$

$$
\begin{aligned}
\left\|\psi C_{\phi} f\right\|_{w, p}^{p} & =\int_{D}|\psi(z)|^{p}|f(\phi(z))|^{p} w(z) \mathrm{d} A(z) \\
& \leq \int_{D} \frac{|\psi(z)|^{p} C}{v(0)\left(1-|\phi(z)|^{2}\right)^{2} v(\phi(z))} w(z)\|f\|_{v, p}^{p} \mathrm{~d} A(z) \\
& \leq \sup _{z \in D} \frac{|\psi(z)|^{p} C}{v(0)\left(1-|\phi(z)|^{2}\right)^{2} v(\phi(z))} w(z)\|f\|_{v, p}^{p}
\end{aligned}
$$


and the claim follows.

Next, we turn our attention to weights $v$ of the form $v=|u|$, where $u$ is a holomorphic function on $D$ without any zeros on $D$. The proof of the following theorem was inspired by the proof of [6, Theorem 1].

Theorem 2 Let $u$ be an analytic function on D without any zeros on D. Put $v(z)=|u(z)|, z \in D$. Moreover let $w$ be an arbitrary weight on $D$ and $\phi$ be an analytic self-map of $D$. Furthermore let $\psi$ be analytic on $D$. Then the weighted composition operator

$$
\psi C_{\phi}: A_{v}^{2} \rightarrow A_{w}^{2}, \quad f \rightarrow \psi(f \circ \phi)
$$

is bounded if and only if the weighted Berezin transform $B_{\phi, v}\left(|\psi|^{2} w\right) \in L^{\infty}(D)$.

Proof. Our proof uses a reformulation of the Carleson measure condition. By definition, $\psi C_{\phi}: A_{v}^{2} \rightarrow$ $A_{w}^{2}$ is bounded if and only if there is $C>0$ such that for every $f \in A_{v}^{2}$ :

$$
\int_{D}|f(\phi(z))|^{2}|\psi(z)|^{2} w(z) \mathrm{d} A(z) \leq C \int_{D}|f(z)|^{2} v(z) \mathrm{d} A(z)
$$

Since $f \in A_{v}^{2}$ if and only if $g=u^{\frac{1}{2}} f \in A_{1}^{2}$ (which means $f=\frac{g}{u^{1 / 2}}$ ), (1) is equivalent to the following condition: There is a constant $C>0$ such that for every $g \in A_{1}^{2}$

$$
\int_{D} \frac{|g(\phi(z))|^{2}}{v(\phi(z))}|\psi(z)|^{2} w(z) \mathrm{d} A(z) \leq C \int_{D}|g(z)|^{2} \mathrm{~d} A(z) .
$$

Let $\mathrm{d} \nu_{v, w, \psi}(z)=|\psi(z)|^{2} \frac{w(z)}{v(\phi(z))} \mathrm{d} A(z)$ and let $\mu_{v, w, \psi}=\nu_{v, w, \psi} \circ \phi^{-1}$ be the pull-back measure induced by $\phi$. If we change variable $s=\phi(z)$, then we get

$$
\int_{D}|g(\phi(z))|^{2}|\psi(z)|^{2} \frac{w(z)}{v(\phi(z))} \mathrm{d} A(z)=\int_{D}|g(\phi(z))|^{2} \mathrm{~d} \nu_{v, w, \psi}(z)=\int_{D}|g(s)|^{2} \mathrm{~d} \mu_{v, w, \psi}(s) .
$$

Thus, (1) is equivalent to $\int_{D}|g(s)|^{2} \mathrm{~d} \mu_{v, w, \psi}(s) \leq C \int_{D}|g(s)|^{2} \mathrm{~d} A(s)$. By Theorem 1 this holds if and only if

$$
\sup _{a \in D} \int_{D}\left|\sigma_{a}^{\prime}(s)\right|^{2} \mathrm{~d} \mu_{v, w, \psi}(s)<\infty
$$

Changing the variable back to $z$, we get

$$
\sup _{a \in D} \int_{D}\left|\sigma_{a}^{\prime}(\phi(z))\right|^{2}|\psi(z)|^{2} \frac{w(z)}{v(\phi(z))} \mathrm{d} A(z)<\infty,
$$

and the claim follows.

\section{References}

[1] Bonet, J., Domański, P. And Lindström, M., (1999). Essential norm and weak compactness of composition operators on weighted Banach spaces of analytic functions, Canad. Math. Bull., 42, 2, 139-148.

[2] Bonet, J., Domański, P. And Lindström, M., (1998). Composition operators between weighted Banach spaces of analytic functions, J. Austral. Math. Soc. (Series A), 64, 101-118.

[3] Bonet, J., Friz, M. And Jordá, E., (2005). Composition operators between weighted inductive limits of spaces of holomorphic functions, Publ. Math., 67, 3-4, 333-348.

[4] Contreras, M. D. And Hernández-Díaz, A. G., (2000). Weighted composition operators in weighted Banach spaces of analytic functions, J. Austral. Math. Soc. (Series A), 69, 41-60. 
[5] Cowen, C. And MacCluer, B., (1995). Composition Operators on Spaces of Analytic Functions, CRC Press, Boca Raton.

[6] ČUČKović, Z. AND ZhaO, R., (2004). Weighted composition operators on the Bergman space, J. London Math. Soc., 70, 2, 499-511.

[7] Duren, P. And Schuster, A., (2004). Bergman spaces, Mathematical Surveys and Monographs 100, American Mathematical Society, Providence, RI.

[8] Hedenmalm, H., Korenblum, B. And Zhu, K., (2000). Theory of Bergman spaces, Graduate Texts in Mathematics, 199, Springer-Verlag, New York.

[9] Kriete, T. And MacCluer, B., (1992). Composition operators on large weighted Bergman spaces, Indiana Univ. Math. J., 41, 3, 755-788.

[10] MacCluer, B., Ohno, S. And ZhaO, R., (2001). Topological structure of the space of composition operators on $H^{\infty}$, Integral equations Operator Theory, 40, 4, 481-494.

[11] Shapiro, J. H., (1993). Composition Operators and Classical Function Theory, Springer.

[12] Sharma, A. K., Sharma, S. D., (2006). Weighted composition operators between Bergman-type spaces, Commun. Korean Math. Soc., 21, 3, 465-474.

\section{Elke Wolf}

Mathematical Institute,

University of Paderborn,

D-33095 Paderborn, Germany.

lichte@math.uni-paderborn. de 